\title{
Correction to: Cooperative disturbance rejection control of vibrating flexible riser systems
}

\author{
Zhijia Zhao C Choon Ki Ahn • Guilin Wen
}

Published online: 13 November 2019

(C) Springer Nature B.V. 2019

\section{Correction to:}

\section{Nonlinear Dyn}

https://doi.org/10.1007/s11071-019-05139-7

The 6th line of equation (49) has been corrected in the original article with $\frac{\chi \rho}{2}+$. The corrected equation is given below:

$$
\begin{aligned}
\dot{S} \leq & -\left(k_{3}-\frac{1}{2}-\frac{\psi E I}{2 k_{2}}\right) u_{i}^{2}+\frac{l}{\delta_{b}} F_{3}^{2}+\frac{1}{\delta_{a}} D_{3}^{2} \\
& -\left(\frac{\psi E I k_{1}^{2}}{2 k_{2}}-\frac{\psi\left|k_{2} T-E I k_{1}\right|}{2 k_{2} \varepsilon_{4}}\right. \\
& \left.-\frac{E I\left|k_{1} \psi-l \chi\right| \varepsilon_{5}}{2}-\frac{\chi T l}{2}\right) x^{\prime 2}(l) \\
& -\left(\frac{\psi E I}{2 k_{2}}-\frac{\psi\left|k_{2} T-E I k_{1}\right| \varepsilon_{4}}{2 k_{2}}-\frac{\chi \rho l}{2}\right) \dot{x}^{2}(l) \\
& -\left(\frac{\psi E I k_{2}}{2}-\frac{E I\left|k_{1} \psi-l \chi\right|}{2 \varepsilon_{5}}\right) x^{\prime \prime \prime 2}(l)
\end{aligned}
$$

The original article can be found online at https://doi.org/10. 1007/s11071-019-05139-7.

\section{Z. Zhao $(\varangle) \cdot$ G. Wen}

School of Mechanical and Electrical Engineering,

Guangzhou University, Guangzhou 510006, China e-mail: zhjzhaoscut@163.com

\section{Z. Zhao $\cdot$ G. Wen}

Advanced Technology Center for Special Equipment,

Guangzhou University, Guangzhou 510006, China

C. K. Ahn $(\bowtie)$

School of Electrical Engineering, Korea University, Seoul

136-701, South Korea

e-mail: hironaka@korea.ac.kr

$$
\begin{aligned}
& -\left(\frac{\chi \rho}{2}+\psi c-\psi \varepsilon_{1}-\frac{l \chi c}{\varepsilon_{2}}\right) \int_{0}^{l} \dot{x}^{2} \mathrm{~d} y \\
& -\left(\frac{\chi T}{2}-\chi \varepsilon_{2} c l-\chi \varepsilon_{3} l\right) \int_{0}^{l} x^{\prime 2} \mathrm{~d} y \\
& -\frac{3 \chi E I}{2} \int_{0}^{l} x^{\prime \prime 2} \mathrm{~d} y \\
& -\int_{0}^{l} \tilde{D}_{2}^{T}\left[-\Upsilon_{2}-\delta_{b} I_{2}-\left(\frac{\psi}{\varepsilon_{1}}+\frac{l \chi}{\varepsilon_{3}}\right) I_{2}\right] \tilde{D}_{2} \mathrm{~d} y \\
& -\tilde{D}_{1}^{T}\left(-\Upsilon_{1}-\delta_{a} I_{2}-0.5 I_{2}\right) \tilde{D}_{1},
\end{aligned}
$$

The original article has been corrected.

Publisher's Note Springer Nature remains neutral with regard to jurisdictional claims in published maps and institutional affiliations. 